\title{
The Sirtuins in Aging and Metabolic Regulation
}

\author{
T. C. LaGuire, S. K. Reaves*
}

Food Science and Nutrition Department, California Polytechnic State University, San Luis Obispo, USA.

Email: *sreaves@calpoly.edu

Received April 22 ${ }^{\text {nd }}, 2013$; revised May 22 ${ }^{\text {nd }}, 2013$; accepted May 30 ${ }^{\text {th }}, 2013$

Copyright (C) 2013 T. C. LaGuire, S. K. Reaves. This is an open access article distributed under the Creative Commons Attribution License, which permits unrestricted use, distribution, and reproduction in any medium, provided the original work is properly cited.

\begin{abstract}
Numerous theories of how and why aging occurs have been postulated but a definitive comprehensive explanation remains elusive. Attempts to unravel genetic details of aging resulted in the identification of a yeast gene known as Sir2 as a modulator of life span. Identification and characterization of mammalian Sir2 homologs followed and has catapulted aging research to exciting new levels. This review begins with basic definitions of aging and then describes some of the most common theories of the aging process. The review presents information related to the initial discovery of the Sirtuins and summarizes some of the recent advances in defining roles for Sirtuin family members. SIRT6 is discussed in greater detail because it is one of the best characterized of the mammalian Sirtuins and seems to be one of the most important in the aging process and metabolic regulation.
\end{abstract}

Keywords: Aging; Sirtuins; SIR-2; SIRT1; SIRT3; SIRT4; SIRT5; SIRT6; NF-kB; Metabolic Regulation

\section{Definition of Aging}

There are various and numerous ways to define aging. Aging is basically defined as the biological impairment of normal function probably as a result of changes made to cells and structural components such as bone and muscle. Aging can also be thought of as the accumulation of changes in a person or organism over time. Biological aging is also sometimes referred to as "senescence" and is considered the changes in the biology of an organism after it reaches maturity. In fact, the word senescence is derived from the Latin word senescere which means "to grow old”. In humans, it is a multidimensional process that includes biological, functional and social change. There are numerous theories as to how and why the changes occur and the study of aging is the focus of much research as scientists attempt to determine if there are ways to slow, avoid or even reverse the effects of aging.

\section{Putative Causes and Theories of Aging}

Because aging potentially affects every human being there is a tremendous amount of research examining the process of aging. As findings have accumulated, a number of models and theories have developed and been tested in an attempt explain aging. Some of the theories *Corresponding author. are based on evolutionary events and involve the accumulation of changes to an organism's makeup over time. Some of the theories of aging involve specific physiologic and or cellular alterations that occur and may explain why an organism such as humans age during their lifetime. A number of the physiologic and cellular theories are identified and briefly explained below.

The telomere theory of aging hypothesizes that normal somatic cells have a finite life span that is determined by the length of their telomeric DNA. Telomeric DNA consists of long repeating sequences that are located at the ends of chromosomal DNA and are added and maintained by an enzyme known as telomerase [1]. Cells typically seem to lose telomeric DNA as they divide and when this DNA reaches a specific shortened length the cells tend to enter senescence and stop dividing. Indeed, this is an intriguing theory as the promotion of telomerase activity and re-lengthening of telomerase activity in human senescent cells allowed the cells to exit sensecence and replicate [2]. Research related to this theory has continued and it is still considered to be a relevant theory of aging.

The autoimmune theory of aging is a fairly recent hypothesis. It states that the body later in life begins to produce autoantibodies against its own tissues and/or there are time-acquired deficits in T-cell function. These changes make the body susceptible to infections, cancer, and 
other chronic diseases especially autoimmune diseases. The role of autoimmune aberrations in aging, like many other theories, remains to be determined. However, there is indeed strong evidence that the immune system is involved in the aging process and this has led to the term "inflamm-aging" to describe the process. In this model, innate immunity seems to be activated and this causes systemic inflammation that alters both systems and cellular processes and these contribute to the aging phenoltype [3]. One of the key molecules in this process appears to be the well-known, well-described transcription factor complex known as Nuclear Factor Kappa-B (NF$\mathrm{kB}$ ). This is one of the most studied theories in aging and will be discussed in greater detail in the subsequent section on NF-kB.

Considerable attention has also been placed on epigenetics and aging. The term epigenetics was coined to explain alterations related to a gene's expression that does not directly alter the nucleotide sequence of the gene. The alterations include structural modifications to chromatin that regulate the transcription of certain genes. These modifications include DNA-methylation, histone acetylation, imprinting, RNA-interference, gene silencing and paramutations [4]. Epigenetics plays a role in regulating numerous genes that have been shown to have roles in aging and shorten lifespan. One of the best examples of how epigenetics is related to aging is the gene in yeast known as Sir-2. Genetic studies showed caloric restriction increased longevity and that Sir-2 was involved and subsequently it was shown that Sir-2 is a member of family of genes now known as the SIRTs (sirtuins) that are expressed in a wide variety of organisms including humans. Mechanistic studies subsequently showed that SIRTs are deacetylases that modify chromatin structure to modulate transcription of genes via epigenetics. The SIRTs and epigenetics are one of the most researched aging theories and will be discussed in greater detail in a later section.

When caloric restriction was first found to increase longevity in rats [5], researchers began to perform follow up studies designed to determine the mechanisms involved. Eventually a theory developed that suggested that perhaps the reason caloric restriction promoted longevity was because fewer free radicals (also known as reactive oxygen species (ROS)) were formed due to less metabolism of energy producing substrates. This is now known as the free radical theory of aging of Harman and it proposes that during aging, macromolecules are oxidized and accumulate to compromise cellular functions and shorten lifespan [6]. The free radicals damage cellular membranes, proteins and DNA, as well as other molecules, and it was hypothesized that the damage to mitochondria was especially harmful and was linked to lifespan [7].
However, recent studies have indicated there are shortcomings to the free radical theory of aging. Nutritional and genetic strategies that increase anti-oxidants typically have not resulted in an increased lifespan. For example, when the results of nineteen clinical trials with vitamin $\mathrm{E}$ supplementation were compiled there appeared to be no reduction in mortality [8]. Supplementation with vitamin $\mathrm{C}$ also appeared to have no significant benefit to lifespan extension [9]. Studies using molecular techniques to increase levels of key anti-oxidant enzymes had mixed results with some having no effect while others increased lifespan of mice [10-12]. Another challenge to the free radical theory of aging is that of exercise. In general, exercise increases free radical production [13] and cellular damage and yet exercise generally enhances human aging [14] and extends lifespan in mice [15] and rats [16]. Overall, there is validity in the free radical theory of aging but it does not account for all aspects of the aging process.

\section{Role of Sirtuins in Aging}

\subsection{Sirtuins and Longevity}

In the late 1980's, a group of genes was first described in the yeast Saccharomyces cerevisiae as having a role in determining yeast mating types. The genes were called the SIR (silent information regulator) genes because they could transcriptionally repress the silent mating loci known as HML and HMR $[17,18]$. Additional roles were also characterized for the SIR genes but in the late 1990's a breakthrough was made that linked one of the SIR genes to increased lifespan (longevity) in yeast and subsequent research into their functions increased exponentially. The breakthrough was in 1999 when Kaeberlein et al. showed that the yeast Sir2 gene decreased life span when it was mutated but increased lifespan when wildtype Sir2 expression was increased [19]. As mentioned previously, years ago a study by McCay et al. (1939) showed that caloric restriction (CR) in rats extended their lifespan [5]. Additional studies in several species and organisms indicated that CR extends their life span as well. A follow-up study, by the same research group that showed Sir2 increased longevity, provided evidence that caloric restriction could not increase longevity in Sir2 mutant yeast. This indicated that Sir2 was indeed required for the increased lifespan with caloric restriction [20]. Subsequent analysis indicated SIRT1 is the mammalian homolog of the yeast Sir2. It has since been determined that mammals express seven homologs of yeast Sir2 and they are known as Sirtuins 1 - 7 [21]. However, it has been shown that SIRT1 has the closest structural and sequence similarity to Sir2, hence it received the most attention in the initial phase of research in mammalian sirtuins. 


\subsection{Characterization of the Sirtuins}

There are now a multitude of details known about the Sirtuins. The Sirtuins are NAD-dependent deacetylases that remove acetyl groups from amino acid residues of histone proteins. Sir2 and SIRT1 were the first in the group to be identified as deacetylases [22]. Acetylation of lysine residues is a common post-translational modification often used in epigenetic regulation. By deacetylating histones the SIRTs alter chromatin structure and modulate expression of genes involved in a wide range of activities and different tissues. However, recent work has indicated that SIRTs also deacetylate molecules that are not histones. For example, SIRT1 deacetylates cytosolic acetyl-CoA synthetase [23], a key enzyme in fatty acid synthesis. SIRT1 also deacetylates several transcription factors including PGC-1alpha [24]. In this process, fasting induces SIRT1 protein in the liver which then interacts with and deacetylates PGC-1alpha which induces gluconeogenic genes and hepatic glucose output. In addition, the deacetylation of PGC-1alpha by SIRT1 caused repression of hepatic glycolysis in fasting [24]. These findings implicate SIRT1 as a key regulator of energy homeostasis in addition to its originally described roles.

Other studies have shown that SIRT1 deacetylates transcription factors that are involved in the mammalian cellular stress response. Findings reported by Brunet et al., 2004 [25], provided evidence that SIRT1 interacted with members of the stress-response FOXO transcription factor family known as Forkhead. Specifically, it was found that SIRT1 and the transcription factor FOXO3 formed a complex when cells were exposed to oxidative stress. When this occurred, SIRT1 deacetylated FOXO3 and increased FOXO3's ability to induce cell cycle arrest. This also inhibited FOXO3's ability to induce cell death. This implies that "one way in which members of the Sir2 family of proteins may increase organismal longevity is by tipping FOXO-dependent responses away from apoptosis and toward stress resistance” [25]. Another intriguing recent finding was that SIRT1 physically binds to a component of NF-kB and inhibits its effects on gene expression [26]. Therefore, perhaps at least one member of the Sirtuin family may enhance longevity by decreasing the inflamm-aging process via NF-kB. These data suggest that the elevation of SIRT1 levels associated with caloric restriction may impart some of its increased longevity effects by decreasing the effects of NF-kB.

Moreover, recent work has also shown beneficial effects of dietary components such as quercetin (a natural polyphenolic flavonoid) and resveratrol (polyphenol found in relatively high amounts in grapes) may exert their effects via activation of SIRT1 $[27,28]$. SIRT1 has also been reported to modulate plasma glucose levels and insulin sensitivity of tissues [29]. There are now significant numbers of studies on how dietary components regulate
SIRT1 and other SIRT family members. In fact, the number of studies is too significant to report in this review. In view of the findings related to the multiple roles of SIRT1 in regulating so many vital processes including lifespan, it seems likely the investigation of ways to manipulate SIRT activity via dietary and lifestyle options will expand dramatically in the near future.

\subsection{Roles of Sirtuin Family Members}

Research is underway to characterize other Sirtuin family members and as a result it has been shown that SIRTs 1 , 2, 6 and 7 are in the nucleus but 3, 4, and 5 are in the mitochondria. SIRT3 is uniquely intriguing because it has been shown to shuttle from the cytosol to the mitochondria during cellular stress [30]. This protein appears to have effects on multiple cellular components including genes in the nucleus and the mitochondria although most of its effects appear to be in mitochondria. For example, a recent study by Ahn et al. (2008) indicated SIRT3 upregulates ATP production by enhancing levels of electron transport chain Complex I components [31]. In addition, it was recently shown that SIRT3 levels significantly decreased with age and were associated with decreased ATP production [32].

Protein acetylation in mitochondria is now believed to be a significant part of regulation of mitochondrial processes [33]. A recent study reported acetylated sites on 195 proteins including a significant number of non-histone proteins. It is of interest that 133 of the proteins were mitochondrial proteins [34]. SIRT3 appears to be the major regulator of mitochondrial acetylation and this is supported by experiments that showed no significant differences in mice lacking SIRT4 and 5 [35] while hyperacetylation was observed in SIRT3 null mice. SIRT3 is also implicated as having a role in the hyperacetylation of mitochondrial protein that occurs in mice with fatty liver due to a chronic high fat diet [36]. In the livers of mice fed a high-fat diet there was a decrease in SIRT3 activity, a 3-fold decrease in NAD+ levels and increased oxidation of mitochondrial proteins. This was associated with reduced activity of electron transport chain Complexes III and IV and this could increase the risk of hepatic lipotoxicity [36]. Therefore it appears that SIRT3 is a key regulator of mitochondrial metabolism and is sensitive to dietary effectors such as a high fat diet.

Although the mechanisms remain largely unexplained, evidence suggests that some of the beneficial effects of CR on longevity involve alterations in mitochondrial functions [37]. Studies have shown that CR tends to increase mitochondrial biogenesis and decrease reactive oxygen species (ROS) in mammals and these responses tend to decrease oxidative damage to macromolecules and cellular damage, two items that are associated with reduced longevity. Because of the vital role acetylation plays in 
mitochondrial function it stands to reason that acetylation is involved in CR and that SIRT3 must play a role. Indeed, it has been shown that SIRT3 increases mitochondrial biogenesis via activation of the transcription factor known as PGC-1alpha [38-40]. The reciprocal is also true as PGC-1alpha stimulates SIRT3 expression by binding to the SIRT3 promoter [41]. PGC-1alpha is a co-activator protein that is responsible for affecting the expression of a large number of genes, in fact so many genes are affected that it is referred to as a master regulator of gene expression and metabolism. Given the apparent connection between SIRT3 and PGC-1alpha there seems no doubt that SIRT3 has the ability to regulate a high number of cellular processes to affect the status and longevity of organisms.

Less is known about the roles of SIRTs 4 and 5 as compared to SIRTs 1 and 3. SIRT4 is present in the mitochondrial matrix and is highly expressed in kidney, heart, brain, liver and pancreatic beta-cells [42,43]. During CR SIRT4 expression declines in the liver but its levels increase in genetic models used to mimic diabetes $[43,44]$. Interestingly, to this point no deacetylase activity has been found for SIRT4 [42,43]. However, it has been shown in mice that SIRT4 ADP-ribosylates glutamate dehydrogenase $(\mathrm{GDH})$ resulting in inactivation of the enzyme [43]. GDH converts glutamate to alpha-ketoglutarate and repression of GDH by SIRT4 decreases the amino acid stimulation of insulin secretion from pancreatic beta-cells [43]. Therefore, reduced SIRT4 activity allows higher GDH activity and amino acid-stimulated insulin secretion during CR when amino acids are being used as an energy source at a higher level. Another recent study has also shown that SIRT4 knockdown in tissue culture and mouse liver caused increased expression of fatty acid metabolism enzymes [44]. Whether or not SIRT4 will be found to have deacetylase activity remains to be determined. However, it is already apparent that SIRT4 plays a role in regulating energy pathways.

SIRT5 appears to differ from SIRTs 3 and 4 in that its liver levels do not seem to change in response to CR $[45,46]$. SIRT5 null mice do not show any major phenotype and there is not a difference in mitochondrial acetylation of proteins [35] as there is in SIRT3 null mice. SIRT5 does not appear to have robust deacetylase activity and does not have high propensity to act upon the typical target of histone proteins $[47,48]$. However, it has been shown that SIRT5 interacts with and deacetylates carbamoyl phosphate synthetase 1 (CPS1) and this activates the enzyme [45]. CPS1 combines ammonia with carbon dioxide in the first, rate-limiting step of the urea cycle in the mitochondria of liver. The study by Nakagawa et al. (2009) showed that SIRT5-deficient mice had elevated levels of ammonia during a prolonged fast due to insufficient up-regulation of CPS1 activity [45]. There- fore, SIRT5 appears to play a key role in the regulation of the urea cycle and ammonia disposal. To date, this is the only known role for SIRT5 but it seems likely additional functions will be uncovered.

\section{SIRT6 in Aging}

\subsection{Defining a Role for SIRT6}

SIRT6 is one of the well-characterized members of the SIRTs family and it has been described as having a role in numerous functions. A few years ago, Michishita et al. (2008) provided evidence that SIRT6 binds to telomere chromatin and can modify its structure by functioning as a NAD-dependent deacetylase [49]. Their work indicated SIRT6 could remove an acetyl group from histone $\mathrm{H} 3$ lysine 9 (H3K9) and that this can modify the structure of the telomeric chromatin. Telomeres are long repeating sequences of DNA that exist on the ends of each of the chromosomes [1,50]. Maintenance of telomere structure has been shown to be vital in protecting against genomic instability and telomere dysfunction can lead to tumor formation [51,52]. Michishita et al. (2008) utilized knockdown experiments and provided evidence that loss of SIRT6 function results in complications similar to Werner Syndrome [49,53]. Additional work by this research group showed that SIRT6 is also involved in H3K56 deacetylation and that this is important for the regulation of cell cycle arrest [54]. Therefore, the last few years have suggested that SIRT6 has an important role in protecting cellular telomeres and cell cycle regulation. In view of these findings, SIRT6 may be important for protection against cancer since dysfunction of telomeres and cell cycle regulation may lead to cancer.

Another theory related to tumorigenesis is that cells must accumulate several DNA mutations as they change from normal cells to cancer cells. Because cells have several DNA repair mechanisms/pathways there is typically an inherent ability to repair DNA before mutations cause cellular transformation to cancerous cells. One of the first studies to link SIRT6 to the DNA repair systems was one using SIRT6 deficient mouse embryonic fibroblasts (MEFs) and stem cells [55]. The investigators reported that SIRT6 deficient cells were more sensitive to DNA damaging agents and they divided more slowly than wild type cells. The data also indicated that SIRT6 was involved in regulating base excision repair (BER), one of the DNA repair pathways [55]. However, at this point the precise role of SIRT6 in BER is yet to be defined.

Another DNA repair mechanism is the repair of double-strand breaks (DSB) in the DNA structure. When DNA contains a mutation that is more significant than a single nucleotide mismatch then a DSB is a typical result. The DSB can then lead to chromosomal end fusion and 
genomic instability. In 2009, McCord et al. showed that SIRT6 participates in DSB DNA repair by forming a macromolecular complex with the DNA DSB repair protein DNA-dependent protein kinase [56]. The interaction with SIRT6 stabilizes the kinase attachment to chromatin and facilitates DSB repair [56]. In 2011 it was reported that oxidative stress leading to DSB causes SIRT6 to translocate to the site of the DNA DSB [57]. These studies suggest an important role for SIRT6 in genomic stability because SIRT6 appears to support DSB repair. SIRT6 was found to associate with Poly-ADP-ribose Polymerase 1 (PARP1) during the conditions of oxidative stress. PARP1 is an enzyme that modifies nuclear proteins by ADP-ribosylation and is involved in a number of processes including DNA repair. The result of SIRT6-PARP1 association was ribosylation of lysine residue 521 of PARP1 and its activation which enhanced DSB repair during the oxidative stress conditions [57]. In summary, it appears SIRT6 is important for DNA repair by both the BER and DSB pathways.

\subsection{SIRT6-Deficient Phenotype}

SIRT6 deficiency appears to have more detrimental effects as compared to deficiencies of the other SIRT family members. Work by Mostoslavsky et al. (2006) described the phenotype of SIRT6 knockout mice as one that leads to numerous abnormalities early in the life cycle [55]. The mice are born with an apparently normal phenotype but it digresses rapidly and at about three weeks of age they develop severe conditions that lead to death at about four weeks of age. Metabolic defects are significant and include low levels of serum IGF-1 and acute onset of hypoglycemia. The SIRT6-deficient mice also exhibit total loss of subcutaneous fat, osteopenia and lymphopenia among documented conditions [55]. Based on the phenotype of SIRT6 knockout mice it is obvious that SIRT6 plays a crucial role in vital processes although the precise relationship between SIRT6 function and each of the observed complications is not yet known.

Recent work using the SIRT6 knockout model has provided important information in defining the role of SIRT6 in glucose homeostasis and how it may relate to lifespan [58]. SIRT6 deficiency results in lethal hypoglycemia at approximately four weeks of age. The hypoglycemia occurs in the context of increased glucose uptake into brown adipose tissue and muscle tissue. Blood insulin levels were low and hepatic gluconeogenesis was elevated suggesting that the pancreas and liver were responding to low blood glucose rather than causing it [58]. Additional experiments showed that SIRT6 deficiency caused elevated mRNA and protein levels of the membrane glucose transporter GLUT1. Moreover, the expression levels of five key glycolytic genes were increased [58]. Chromatin imm-unoprecipitation (ChIP) assays in- dicated that SIRT6 bound to the promoter regions of the five glycolytic genes and that SIRT6 deficiency increased histone H3K9 acetylation of the promoters of these genes [58]. Because the hypoxia-inducible transcription factor Hif $1 \alpha$ is an important regulator of enhanced glucose utilization under certain conditions [59,60], investigators have sought to determine if there was an interaction between Hif1 $\alpha$ and SIRT6. Co-immunoprecipitation assays provided evidence that there was an interaction between the two proteins and that SIRT6 normally represses the activity of Hif1 $\alpha$ [58]. Further experimentation revealed that SIRT6 relies upon its interaction with Hif1 $\alpha$ in an important regulatory mechanism to control glucose metabolism [58]. These data indicate that SIRT6 functions as a H3K9 deacetylase to regulate glucose metabolism and suggests that methods to regulate SIRT6 activity may be effective in attenuating harmful effects of diabetes, obesity and aging.

\subsection{SIRT6 Overexpression and Effects on Metabolism and Lifespan}

Researchers recently found that male transgenic mice overexpressing SIRT6 had a significantly longer lifespan than wild-type (WT) mice [61]. Interestingly, there was not enhanced lifespan in the female transgenic mice of the same study. Researchers used two transgenic mouse lines made from two different founders to substantiate that findings were not due to a genetic aberration from the transgenic method. The enhanced lifespan in male mice was present in both transgenic lines. The mean lifespan of SIRT6-transgenic relative to WT mice was increased by $14.8 \%$ and $16.9 \%$ in the two SIRT6 transgenic lines compared to WT. The reason for the longer lifespan in SIRT6 enhanced mice was not easily explained although the authors reported intriguing data and potential mechanisms [61]. Research has indicated that SIRT6 plays a crucial role in maintaining genetic stability [55] and because malignant tumors are a common cause of death in mice it is important to investigate tumor numbers in mice overexpressing SIRT6. Examinations by Kanfi et al. (2012) showed no significant differences between WT and transgenic mice in the tumor spectrum or incidence [61]. It was also found that SIRT6 overexpression had a positive effect on blood glucose regulation but this effect was not sex-specific [61]. Further analysis revealed significant differences in metabolic signaling molecules and pathways between WT and SIRT6 transgenic mice. IGF1 levels were significantly different between male WT and transgenic mice but not between female mice and these differences coincided with differences in expression levels of IGFBP1 [61], the molecule believed to be the main acute effector of IGF1 levels [62]. Kanfi et al. also examined IGF1 signaling in some tissues and found that the levels of phosphorylated AKT, 
FOXO1, FOXO3 and IGF receptor were significantly lower in the white adipose tissue of male but not female SIRT6 transgenic mice [61]. These differences in IGF1related molecules are especially intriguing given the relevance of IGF1 in the aging process $[63,64]$. Although these findings do not explain the precise role of SIRT6 in aging it does suggest specific aspects that need to be further examined.

The current epidemic of obesity and metabolic syndrome compels investigations into whether anti-aging factors such as SIRT6 may be protective even in the context of obesity. To explore these interrelationships, a recent study examined the effects of SIRT6 over-expression in mice in the context of diet-induced obesity [65]. WT and SIRT6 transgenic mice were fed a high-fat diet (HFD) for 16 weeks that contained $60 \%$ of calories from fat. In addition, separate groups of WT and SIRT6 transgenic mice that consumed standard chow served as controls. No significant differences between any of the four groups were found for food intake or body weight. However, when fed with HFD the SIRT6 transgenic mice had significantly lower total body fat and higher lean body mass than their WT littermates [65]. When fed HFD, SIRT6 transgenic mice also had lower serum triglycerides (33\% lower), lower LDL-cholesterol (30\% lower) and improved glucose uptake during glucose tolerance testing. Transcription profile analysis indicated that the SIRT6 transgenic mice had lower levels of angiopoietin-like protein 4 (ANGPTL4) and DGAT1 as compared to their WT littermates [65]. ANGPTL4 negatively regulates lipoprotein lipase [66], the enzyme that hydrolyzes triglycerides from plasma lipoproteins so a decrease in ANGPTL4 activity caused by elevated SIRT6 could be a mechanism that causes a decrease in plasma triglycerides. DGAT1 is an intracellular enzyme that is involved in triglyceride synthesis [67] and the 50\% decrease in DGAT1 expression in fat tissue of SIRT6 transgenic mice would be expected to significantly decrease triglyceride synthesis [65]. Researchers were also able to determine that SIRT6 directly binds to the DGAT1 promoter and that there was a 3.5-fold higher level of SIRT6 bound in the transgenic mice as compared to their WT littermates [65]. These data suggest SIRT6 is an important repressor of DGAT1 expression. Overall, results from the work of Kanfi et al. (2010) demonstrate SIRT6 overexpression is protective against HFD-induced damage such as lipotoxicity, increased body fat storage and aberrations in regulation of glucose homeostasis [65].

\subsection{SIRT6 and NF-kB}

The family of transcription factors known as NF-kB has also been implicated in the aging process. One of the key components of NF-kB is the factor known as RELA or p65 because it has been shown to affect the regulation of a variety of genes. A recent study provided evidence of a direct relationship between NF-kB and SIRT6 when it was shown that RELA interacts physically with SIRT6 thereby suggesting that these factors cooperate in regulating certain processes [68]. Researchers used immunoprecipitates (IPs) from 293T cells expressing FLAGtagged SIRT6 and Western blot analyses to show SIRT6 binds RELA and it appears specific to SIRT6 as compared to other SIRT members. When IPs were done using endogenous proteins it was also shown that RELA binds to SIRT6 preferentially over other SIRTs [68]. Chromatin immunoprecipitation (ChIP) analysis indicated TNF- $\alpha$ enhanced levels of SIRT6 bound to promoters of RELA target genes and that this resulted from RELA recruiting SIRT6 to the promoters of genes affected by RELA [68]. Subsequent experiments provided evidence that SIRT6 directly inhibits expression of certain NF-kB target genes and that the mechanism appears to be via SIRT6-directed deacetylation of histone H3 lysine 9 residues that destabilizes RELA binding to chromatin [68]. To validate these findings, it was also shown that SIRT6 depletion led to increased expression of NF$\mathrm{kB}$ target genes and RELA deficiency via heterozygosity improved the phenotype of SIRT6 deficient mice [68]. Taken together, these findings point out a direct interaction of SIRT6 and NF-kB that has significant health implications. Although the precise role of this interaction remains to be fully defined [69], its potential importance to health and wellbeing makes it worthy of future research efforts.

Recent work has also focused on the relationship of SIRT6 and NF-kB and their genome-wide effects on gene expression. A recent study utilized genome-scale ChIP-chip assays with oligonucleotide arrays to characterize the binding of SIRT6 and RELA in mouse embryonic fibroblasts [70]. The assays were done before and after stimulation with TNF- $\alpha$ to examine effects of the stress response of these two protein factors. Results indicated that altogether SIRT6 can bind up to approximately 5050 gene promoters including 1899 genes in unstimulated cells. Upon TNF- $\alpha$ stimulation there was a significant and dynamic movement of SIRT6 from some promoters to others within minutes following TNF- $\alpha$ addition [70]. Analyses also showed that RELA bound to 2738 promoters. Assays revealed that on 1481 promoters both SIRT6 and RELA bound and that RELA plays a significant role in SIRT6 localizing to certain gene promoters [70]. These findings provided more evidence of the cooperation of these two factors in regulating numerous genes in controlling cellular responses.

In summary, there is a great deal more that needs to be uncovered and explained about the aging process. The fairly recent discovery of the Sirtuin family members has provided important insight into the aging process and 
appears to be a promising avenue for future studies. These efforts should include how diet and lifestyle may influence the Sirtuins as a means of extending lifespan and improving quality of health.

\section{REFERENCES}

[1] S. E. Artandi, “Telomeres, Telomerase, and Human Disease,” New England Journal of Medicine, Vol. 355, No. 12, 2006, pp. 1195-1197. doi:10.1056/NEJMp068187

[2] H. Vaziri and S. Benchimol, "Reconstitution of Telomerase Activity in Normal Human Cells Leads to Elongation of Telomeres and Extended Replicative Life Span,” Current Biology, Vol. 8, No. 5, 1998, pp. 279-282. doi:10.1016/S0960-9822(98)70109-5

[3] A. Salminen, J. Huuskonen, J. Ojala, A. Kauppinen, K. Kaarniranta and T. Suuronen, "Activation of Innate Immunity System during Aging: NF-kB Signaling Is the Molecular Culprit of Inflamm-Aging," Ageing Research Reviews, Vol. 7, No. 2, 2008, pp. 83-105. doi:10.1016/j.arr.2007.09.002

[4] D. C. Allis, T. Jenuwein, D. Reinberg and M.-L. Caparros, "Epigenetics," Cold Spring Harbor Laboratories Press, New York, 2007.

[5] C. M. McCay, L. A. Maynard, G. Sperling and L. L. Barnes, "Retarded Growth, Lifespan, Ultimate Body Size and Age Changes in the Albino Rat after Feeding Diets Restricted in Calories," Journal of Nutrition, Vol. 18, 1939, pp. 1-13.

[6] D. Harman, "Free Radical Theory of Aging: Effect of Free Radical Reaction Inhibitors on the Mortality Rate of Male LAF Mice,” Journal of Gerontology, Vol. 23, No. 4, 1968, pp. 476-482. doi:10.1093/geronj/23.4.476

[7] D. Harman, "Free Radical Theory of Aging: An Update," Annual of the New York Academy of Science, Vol. 1067, 2006, pp. 10-21. doi:10.1196/annals.1354.003

[8] E. R. Miller III, R. Pastor-Barriuso, D. Dalal, R. A. Riemersma, L. J. Appel and E. Guallar, "Meta-Analysis: HighDosage Vitamin E Supplementation May Increase Allcause Mortality,” Annals of Internal Medicine, Vol. 142, No. 1, 2005, pp. 37-46.

doi:10.7326/0003-4819-142-1-200501040-00110

[9] G. Bjelakovic, D. Nikolova, L. L. Gluud, R. G. Simonetti and C. Gluud, "Mortality in Randomized Trials of Antioxidant Supplements for Primary and Secondary Prevention: Systematic Review and Meta-Analysis,” The Journal of American Medical Association, Vol. 297, No. 8, 2007, pp. 842-857. doi:10.1001/jama.297.8.842

[10] V. I. Perez, R. H. Van, A. Bokov, C. J. Epstein, J. Vijg and A. Richardson, "The Overexpression of Major Antioxidant Enzymes Does Not Extend the Lifespan of Mice," Aging Cell, Vol. 8, No. 1, 2009, pp. 73-75. doi:10.1111/j.1474-9726.2008.00449.X

[11] A. Mitsui, J. Hamuro, H. Nakamura, N. Kondo, Y. Hirabayashi, S. Ishizaki-Koizumi, T. Hirakawa, T. Inoue and J. Yodoi, "Overexpression of Human Thioredoxin in Transgenic Mice Controls Oxidative Stress and Life Span," Antioxidants and Redox Signaling, Vol. 4, No. 4, 2004, pp. 693-696. doi:10.1089/15230860260220201

[12] S. E. Schriner, N. J. Linford, G. M. Martin, P. Treuting, C. E. Ogburn, M. Emond, P. E. Coskun, W. Ladiges, N. Wolf, H. Van Remmen, D. C. Wallace and P. S. Rabinovitch, "Extension of Murine Lifespan by Overexpression of Catalase Targeted to Mitochondria,” Science, Vol. 308, No. 5730, 2005, pp. 1909-1911. doi:10.1126/science.1106653

[13] S. K. Powers and M. J. Jackson, "Exercise-Induced Oxidative Stress: Cellular Mechanisms and Impact on Muscle Force Production,” Physiological Reviews, Vol. 88, No. 4, 2008, pp. 1243-1276. doi:10.1152/physrev.00031.2007

[14] E. Nakamura, T. Moritani and A. Kanetaka, "Effects of Habitual Physical Exercise on Physiological Age in Men Aged 20 - 85 Years as Estimated Using Principal Component Analysis," European Journal of Applied Physiology and Occupational Physiology, Vol. 73, No. 5, 1996, pp. 410-418. doi:10.1007/BF00334417

[15] A. Navarro, C. Gomez, J. M. Lopez-Cepero and A. Boveris, "Beneficial Effects of Moderate Exercise on Mice Aging: Survival, Behavior, Oxidative Stress, and Mitochondrial Electron Transfer," American Journal of Physiology Regulatory, Integrative and Comparative Physiology, Vol. 286, No. 3, 2004, pp. R505-R511. doi:10.1152/ajpregu.00208.2003

[16] J. O. Holloszy, E. K. Smith, M. Vining and S. Adams, "Effect of Voluntary Exercise on Longevity of Rats," Journal of Applied Physiology, Vol. 59, No. 3, 1985, pp. 826831.

[17] J. M. Ivy, A. J. Klar and J. B. Hicks, “Cloning and Characterization of Four SIR Genes of Saccharomyces Cerevisiae," Molecular and Cellular Biology, Vol. 6, No. 2, 1986, pp. 688-702.

[18] J. Rine and I. Herskowitz, "Four Genes Responsible for a Position Effect on Expression from HML and HMR in Saccharomyces Cerevisiae," Genetics, Vol. 116, No. 1, 1987, pp. 9-22.

[19] M. Kaeberlein, M. McVey and L. Guarente, “The SIR2/3/ 4 Complex and SIR2 Alone Promote Longevity in Saccharomyces Cerevisiae by Two Different Mechanisms," Genes Development, Vol. 13, No. 19, 1999, pp. 25702580. doi:10.1101/gad.13.19.2570

[20] S. J. Lin, P. A. Defossez and L. Guarente, "Requirement of NAD and SIR2 for Life-Span Extension by Calorie Restriction in Saccharomyces Cerevisiae," Science, Vol. 289, No. 5487, 2000, pp. 2126-2128. doi:10.1126/science.289.5487.2126

[21] Taylor, et al., "Biological and Potential Therapeutic Roles of Sirtuin Deacetylases," Cellular and Molecular Life Sciences, Vol. 65, No. 24, 2008, pp. 4000-4018. doi:10.1007/s00018-008-8357-y

[22] S. Imai, C. M. Armstrong, M. Kaeberlein and L. Guarente, "Transcriptional Silencing and Longevity Protein Sir2 Is an NAD-Dependent Histone Deacetylase,” Nature, Vol. 403, No. 6771, 2000, pp. 795-800. doi:10.1038/35001622

[23] W. C. Hallows, S. Lee and J. M. Denu, "Sirtuins Deacetylate and Activate Mammalian Acetyl-CoA Synthetases," Proceedings of National Academy Science of the USA, Vol. 103, No. 27, 2006, pp. 10230-10235. 
doi:10.1073/pnas.0604392103

[24] J. T. Rodgers, C. Lerin, W. Haas, S. P. Gygi, B. M. Spiegelman and P. Puigserver, "Nutrient Control of Glucose Homeostasis through a Complex of PGC-1alpha and SIRT1,” Nature, Vol. 434, No. 7029, 2005, pp. 113-118. doi:10.1038/nature03354

[25] A. Brunet, L. B. Sweeney, J. F. Sturgill, K. F. Chua, P. L. Greer, Y. Lin, H. Tran, S. E. Ross, R. Mostoslavsky, et al., "Stress-Dependent Regulation of FOXO Transcription Factors by the SIRT1 Deacetylase,” Science, Vol. 303, No. 5666, 2004, pp. 2011-2015. doi:10.1126/science.1094637

[26] F. Yeung, J. Hoberg, C. Ramsey, M. Keller, D. Jones, R. Frye and M. Mayo, "Modulation of NF-kappaB-Dependent Transcription and Cell Survival by the SIRT1 Deacetylase,” EMBO Journal, Vol. 23, No. 12, 2004, pp. 23692380. doi:10.1038/sj.emboj.7600244

[27] J. M. Davis, E. A. Murphy, M. D. Carmichael and B. Davis, "Quercetin Increases Brain and Muscle Mitochondrial Biogenesis and Exercise Tolerance," American Journal Physiology (Regulation and Integration of Comparative Physiology), Vol. 296, No. 4, 2009, pp. R1071R1077.

[28] M. Knutson and C. Leeuwenburgh, "Resveratrol and Novel Potent Activators of SIRT1: Effects on Aging and Age-Related Diseases," Nutrition Reviews, Vol. 66, No. 10, 2008, pp. 591-596. doi:10.1111/j.1753-4887.2008.00109.x

[29] Metoyer and Pruitt, "The Role of Sirtuin Proteins in Obesity,” Pathophysiology, Vol. 15, No. 2, 2008, pp. 103-108. doi:10.1016/j.pathophys.2008.04.002

[30] M. B. Scher, A. Vaquero and D. Reinberg, "SirT3 Is a Nuclear NAD+-Dependent Histone Deacetylase That Translocates to the Mitochondria upon Cellular Stress," Genes Development, Vol. 21, No. 8, 2007, pp. 920-928. doi:10.1101/gad.1527307

[31] Ahn, et al., "A Role for the Mitochondrial Deacetylase sirt3 in Regulating Energy Homeostasis,” Proceedings National Academy of Sciences, Vol. 105, No. 38, 2008, pp. 14447-14452. doi:10.1073/pnas.0803790105

[32] I. Lanza, D. Short, K. Short, S. Raghavakaimal, R. Basu, M. Joyner, J. McConnel and K. S. Nair, "Endurance Exercise as a Countermeasure for Aging,” Diabetes, Vol. 57, No. 11, 2008, pp. 2933-2942. doi:10.2337/db08-0349

[33] S. Zhao, W. Xu, W. Jiang, W. Yu, Y. Lin, T. Zhang, J. Yao, L. Zhou, Y. Zeng, H. Li, Y. Li, J. Shi, W. An, S. M. Hancock, F. He, L. Qin, J. Chin, P. Yang, X. Chen, Q. Lei, Y. Xiong and K. L. Guan, "Regulation of Cellular Metabolism by Protein Lysine Acetylation,” Science, Vol. 327, No. 3968, 2010, pp. 1000-1004. doi:10.1126/science.1179689

[34] S. C. Kim, R. Sprung, Y. Chen, Y. Xu, H. Ball, J. Pei, T. Cheng, Y. Kho, H. Xiao, L. Xiao, N. V. Grishin, M. White, X. J. Yang and Y. Zhao, "Substrate and Functional Diversity of Lysine Acetylation Revealed by a Proteomics Survey,” Molecular Cell, Vol. 23, No. 4, 2006, pp. 607-618. doi:10.1016/j.molcel.2006.06.026

[35] D. B. Lombard, F. W. Alt, H. L. Cheng, J. Bunkenborg, R. S. Streeper, R. Mostoslavsky, J. Kim, G. Yancopoulos, D.
Valenzuela, A. Murphy, et al., "Mammalian Sir2 Homolog SIRT3 Regulates Global Mitochondrial Lysine Acetylation,” Molecular and Cellular Biology, Vol. 27, No. 24, 2007, pp. 8807-8814. doi:10.1128/MCB.01636-07

[36] A. A. Kendrick, M. Choudhury, S. M. Rahman, C. E. McCurdy, M. Friederich, J. L. K. Van Hove, P. A. Watson, N. Birdsey, J. Bao, D. Gius, M. N. Sack, E. Jing, C. R. Kahn, J. E. Friedman and K. R. Jonscher, "Fatty Liver Is Associated with Reduced SIRT3 Activity and Mitochondrial Protein Hyperacetylation,” Biochemical Journal, Vol. 433, No. 3, 2011, pp. 505-514. doi:10.1042/BJ20100791

[37] R. M. Anderson, D. Shanmuganayagan and R. Weindruch, "Caloric Restriction and Aging: Studies in Mice and Monkeys,” Toxicologic Pathology, Vol. 37, No. 1, 2008, pp. 47-51. doi:10.1177/0192623308329476

[38] O. M. Palacios, J. J. Carmona, S. Michan, K. Y. Chen, Y. Manabe, J. L. Ward 3rd, L. J. Goodyear and Q. Tong, "Diet and Exercise Signals Regulate SIRT3 and Activate AMPK and PGC-1alpha in Skeletal Muscle," Aging ( $\mathrm{Al}$ bany NY), Vol. 1, No. 9, 2009, pp. 771-783.

[39] V. B. Pillai, N. R. Sundaresan, G. Kim, M. Gupta, S. B. Rajamohan, J. B. Pillai, S. Samant, P. V. Ravindra, A. Isbatan and M. P. Gupta, "Exogenous NAD Blocks Cardiac Hypertrophic Response via Activation of the SIRT3LKB1-AMP-Activated Kinase Pathway,” The Journal of Biological Chemistry, Vol. 285, No. 5, 2010, pp. 31333144. doi:10.1074/jbc.M109.077271

[40] T. Shi, F. Wang, E. Stieren and Q. Tong, "SIRT3, a Mitochondrial Sirtuin Deacetylase, Regulates Mitochondrial Function and Thermogenesis in Brown Adipocytes," The Journal of Biological Chemistry, Vol. 280, No. 14, 2005, pp. 13560-13567. doi:10.1074/jbc.M414670200

[41] X. Kong, R. Wang, Y. Xue, X. Liu, H. Zhang, Y. Chen, F. Fang and Y. Chang, " Sirtuin 3, a New Target of PGC1alpha, Plays an Important Role in the Suppression of ROS and Mitochondrial Biogenesis,” PLoS ONE, Vol. 5, No. 7, 2010, Article ID: e11707. doi:10.1371/journal.pone.0011707

[42] N. Ahuja, B. Schwer, S. Carobbio, D. Waltregny, B. J. North, V. Castronovo, P. Maechler and E. Verdin, "Regulation of Insulin Secretion by $\mathrm{SIRT}_{4}$, a Mitochondrial ADP-Ribosyltransferase,” The Journal of Biological Chemistry, Vol. 282, No. 46, 2007, pp. 33583-33592. doi:10.1074/jbc.M705488200

[43] M. C. Haigis, R. Mostoslavsky, K. M. Haigis, K. Fahie, D. C. Christodoulou, A. J. Murphy, D. M. Valenzuela, G. D. Yancopoulos, M. Karow, G. Blander, C. Wolberger, T. A. Prolla, R. Weindruch, F. W. Alt and L. Guarente, "SIRT4 Inhibits Glutamate Dehydrogenase and Opposes the Effects of Calorie Restriction in Pancreatic Beta Cells," Cell, Vol. 126, No. 5, 2006, pp. 941-954. doi:10.1016/j.cell.2006.06.057

[44] N. Nasrin, X. Wu, E. Fortier, Y. Feng, O. C. Bare, S. Chen, X. Ren, Z. Wu, R. S. Streeper and L. Bordone, "SIRT4 Regulates Fatty Acid Oxidation and Mitochondrial Gene Expression in Liver and Muscle Cells," The Journal of Biological Chemistry, Vol. 285, No. 42, 2010, pp. 31995-32002. doi:10.1074/jbc.M110.124164

[45] T. Nakagawa, D. J. Lomb, M. C. Haigis and L. Guarente, 
"SIRT5 Deacetylates Carbamoyl Phosphate Synthetase1 and Regulates the Urea Cycle,” Cell, Vol. 137, No. 3, 2009, pp. 560-570. doi:10.1016/j.cell.2009.02.026

[46] B. Schwer, M. Eckersdorff, Y. Li, J. C. Silva, D. Fermin, M. V. Kurtev, C. Giallourakis, M. J. Comb, F. W. Alt and D. B. Lombard, "Calorie Restriction Alters Mitochondrial Protein Acetylation,” Aging Cell, Vol. 8, No. 5, 2009, pp. 604-606. doi:10.1111/j.1474-9726.2009.00503.x

[47] J. C. Black, A. Mosley, T. Kitada, M. Washburn and M. Carey, "The SIRT2 Deacetylase Regulates Autoacetylation of p300,” Molecular Cell, Vol. 32, No. 3, 2008, pp. 449-455. doi:10.1016/j.molcel.2008.09.018

[48] C. Schlicker, M. Gertz, P. Papatheodorou, B. Kachholz, C. F. Becker and C. Steegborn, "Substrates and Regulation Mechanisms for the Human Mitochondrial Sirtuins Sirt3 and Sirt5,” Journal Molecular Biology, Vol. 382, No. 3, 2008, pp. 790-801. doi:10.1016/j.jmb.2008.07.048

[49] E. Michishita, et al., "SIRT6 Is a Histone H3 Lysine 9 Deacetylase That Modulates Telomeric Chromatin," $\mathrm{Na}$ ture, Vol. 452, No. 7186, 2008, pp. 492-496. doi:10.1038/nature06736

[50] B. McClintock, "The Stability of Broken Ends of Chromosomes in Zea mays," Genetics, Vol. 26, No. 2, 1941, pp. 234-282.

[51] D. Gisselsson, et al., "Telomere Dysfunction Triggers Extensive DNA Fragmentation and Evolution of Complex Chromosome Abnormalities in Human Malignant Tumors," Proceedings of the National Academy of Sciences of the United State of America, Vol. 98, No. 22, 2001, pp. 12683-12688. doi:10.1073/pnas.211357798

[52] A. K. Meeker, et al., "Telomere Length Abnormalities Occur Early in the Initiation of Epithelial Carcinogenesis," Clinical Cancer Research, Vol. 10, No. 10, 2004, pp. 3317-3326. doi:10.1158/1078-0432.CCR-0984-03

[53] A. S. Multani and S. Chang, "WRN at Telomeres: Implications for Aging and Cancer," Journal of Cell Science, Vol. 120, No. 5, 2007, pp. 713-721. doi:10.1242/jcs.03397

[54] E. Michishita, et al., “ Cell Cycle-Dependent Deacetylation of Telomeric Histone H3 Lysine K56 by Human SIRT6,” Cell Cycle, Vol. 8, No. 16, 2009, pp. 2664-2666. doi:10.4161/cc.8.16.9367

[55] R. Mostoslavsky, K. F. Chua, D. B. Lombard, W. W. Pang, M. R. Fischer, L. Gellon, P. Liu, G. Mostoslavsky, S. Franco, M. M. Murphy, K. D. Mills, P. Patel, J. T. Hsu, A. L. Hong, E. Ford, H. L. Cheng, C. Kennedy, N. Nunez, R. Bronson, D. Frendewey, W. Auerbach, D. Valenzuela, M. Karow, M. O. Hottiger, S. Hursting, J. C. Barrett, L. Guarente, R. Mulligan, B. Demple, G. D. Yancopoulos and F. W. Alt, "Genomic Instability and Aging-Like Phenotype in the Absence of Mammalian SIRT6," Cell, Vol. 124, No. 2, 2006, pp. 315-329. doi:10.1016/j.cell.2005.11.044

[56] R. A. McCord, et al., "SIRT6 Stabilizes DNA-Dependent Protein Kinase at Chromatin for DNA Double-Strand Break Repair," Aging (Albany NY), Vol. 1, No. 1, 2009, pp. 109-121

[57] Z. Mao, et al., "SIRT6 Promotes DNA Repair under
Stress by Activating PARP1,” Science, Vol. 332, No. 6036, 2011, pp. 1443-1446. doi:10.1126/science.1202723

[58] L. Zho, A. D’Urso, D. Toiber, C. Sebastian, R. E. Henry, D. D. Vadysirisack, A. Guimaraes, B. Marinelli, J. D. Wikstrom, T. Nir, C. B. Clish, B. Vaitheesvaran, O. Iliopoulos, I. Kurland, Y. Dor, R. Weissleder, O. S. Shirihai, L. W. Ellisen, J. M. Espinosa and R. Mostoslavsky, “The Histone Deacetylase Sirt6 regUlates Glucose Homeostasis via Hif1 $\alpha$," Cell, Vol. 140, No. 2, 2010, pp. 280-293. doi:10.1016/j.cell.2009.12.041

[59] J. J. Lum, T. Bui, M. Gruber, J. D. Gordan, R. J. DeBerardinis, K. L. Covello, M. C. Simon and C. B. Thompson, "The Transcription Factor HIF-1alpha Plays a Critical Role in the Growth Factor-Dependent Regulation of Both Aerobic and Anaerobic Glycolysis," Genes Development, Vol. 21, No. 9, 2007, pp. 1037-1049. doi:10.1101/gad.1529107

[60] T. N. Seagroves, H. E. Ryan, H. Lu, B. G. Wouters, M. Knapp, P. Thibault, K. Laderoute and R. S. Johnson, "Transcription Factor HIF-1 Is a Necessary Mediator of the Pasteur Effect in Mammalian Cells," Molecular and Cellular Biology, Vol. 21, No. 10, 2001, pp. 3436-3444. doi:10.1128/MCB.21.10.3436-3444.2001

[61] Y. Kanfi, S. Naiman, G. Amir, V. Peshti, G. Zinman, L. Nahum, Z. Bar-Joseph and H. Y. Cohen, "The Sirtuin SIRT6 Regulates Lifespan in Male Mice,” Nature, Vol. 483, No. 7388, 2012, pp. 218-221. doi:10.1038/nature10815

[62] P. D. Lee, L. C. Giudice, C. A. Conover and D. R. Powell, "Insulin-Like Growth Factor Binding Protein-1: Recent Findings and New Directions," Proceedings of the Society for Experimental Biology and Medicine, Vol. 216, No. 3, 1997, pp. 319-357.

[63] C. J. Kenyon, “The Genetics of Ageing," Nature, Vol. 464, No. 7288, 2010, pp. 504-512. doi:10.1038/nature08980

[64] M. Holzenberger, et al., "IGF-1 Receptor Regulates Lifespan and Resistance to Oxidative Stress in Mice," Nature, Vol. 421, No. 6919, 2003, pp. 182-187. doi:10.1038/nature01298

[65] Y. Kanfi, V. Peshti, R. Gil, et al., "SIRT6 Protects against Pathological Damage Caused by Diet-Induced Obesity," Aging Cell, Vol. 9, No. 2, 2010, pp. 162-173.

[66] K. Yoshida, T. Shimizugawa, M. Ono and H. Furukawa, "Angiopoietinlike Protein 4 Is a Potent HyperlipidemiaInducing Factor in Mice and Inhibitor of Lipoprotein Lipase,” Journal of Lipid Research, Vol. 43, No. 11, 2002, pp. 1770-1772. doi:10.1194/jlr.C200010-JLR200

[67] S. Cases, S. J. Smith, Y. W. Zheng, H. M. Myers, S. R. Lear, E. Sande, S. Novak, C. Collins, C. B. Welch, A. J. Lusis, S. K. Erickson and R. V. Farese Jr., "Identification of a Gene Encoding an Acyl CoA: Diacylglycerol Acyltransferase, a Key Enzyme in Triacylglycerol Synthesis," Proceedings of the National Academy of Sciences of the United State of America, Vol. 95, No. 22, 1998, pp. 13018-13023. doi:10.1073/pnas.95.22.13018

[68] T. L. Kawahara, E. Michishita, A. S. Adler, M. Damian, E. Berber, M. Lin, R. A. McCord, K. C. Ongaigui, L. D. Boxer, H. Y. Chang and K. F. Chua, "SIRT6 Links His- 
tone H3 Lysine 9 Deacetylation to NF- $\kappa$ B-Dependent gene Expression and Organismal Life Span,” Cell, Vol. 136, No. 1, 2009, pp. 62-74. doi:10.1016/j.cell.2008.10.052

[69] G. Natoli, "When Sirtuins and NF- $\kappa$ B Collide," Cell, Vol. 136, No. 1, 2009, pp. 19-21. doi:10.1016/j.cell.2008.12.034

[70] T. L. A. Kawahara, N. A. Rapicavoli, A. R. Wu, K. Qu, S. R. Quake, et al., "Dynamic Chromatin Localization of Sirt6 Shapes Stress- and Aging-Related Transcriptional Networks,” PLoS Genetics, Vol. 7, No. 6, 2011, Article ID: e1002153. doi:10.1371/journal.pgen.1002153 